\title{
Radio/X-ray correlations and variability in the X-ray binary LS I +61 ${ }^{\circ} 303$
}

\author{
R. Sharma, ${ }^{1 \star}$ M. Massi, ${ }^{1}$ M. Chernyakova, ${ }^{2,3}$ D. Malyshev, ${ }^{4}$ Y. C. Perrott, ${ }^{5,6}$ A. Kraus ${ }^{1}$ \\ S. A. Dzib, ${ }^{1}$ F. Jaron ${ }^{7}$ and T. M. Cantwell ${ }^{8}$ \\ ${ }^{1}$ Max-Planck-Institut für Radioastronomie, Auf dem Hügel 69, D-53121 Bonn, Germany \\ ${ }^{2}$ School of Physical Sciences and C-fAR, Dublin City University, Dublin 9, Ireland \\ ${ }^{3}$ Dublin Institute for Advanced Studies, 31 Fitzwilliam Place, Dublin 2, Ireland \\ ${ }^{4}$ Institut für Astronomie und Astrophysik Tübingen, Universität Tübingen, Sand 1, D-72076 Tübingen, Germany \\ ${ }^{5}$ Astrophysics Group, Cavendish Laboratory, JJ Thomson Avenue, Cambridge CB3 OHE, UK \\ ${ }^{6}$ School of Chemical and Physical Sciences, Victoria University of Wellington, PO Box 600, Wellington 6140, New Zealand \\ ${ }^{7}$ Department of Space, Earth and Environment, Onsala Space Observatory, Chalmers University of Technology, Onsala, Sweden \\ ${ }^{8}$ JBCA, Dept. of Physics $\mathcal{E}$ Astronomy, University of Manchester, Manchester M13 9PL, UK
}

Accepted: 2020 November 9; in original form 2020 August 31

\begin{abstract}
The high-mass X-ray binary LS I $+61^{\circ} 303$ exhibits variability in its radio and X-ray emissions, ranging from minute to hour time-scales. At such short time-scales, not much is known about the possible correlations between these two emissions from this source, which might offer hints to their origin. Here, we study the relationship between these emissions using simultaneous X-ray and radio monitoring. We present new radio observations using the Arcminute Microkelvin Imager Large Array telescope at two frequency bands, 13-15.5 and 15.5-18 GHz. We also describe new X-ray observations performed using the XMM-Newton telescope. These X-ray and radio observations overlapped for five hours. We find for the first time that the radio and X-ray emission are correlated up to 81 per cent with their few percent variability correlated up to 40 per cent. We discuss possible physical scenarios that produces the observed correlations and variability in the radio and X-ray emission of LS I $+61^{\circ} 303$.
\end{abstract}

Key words: Radio continuum: stars - X-rays: binaries $-\mathrm{X}$-rays: individual: LS I $+61^{\circ} 303-$ stars:jets - radiation mechanisms:general - magnetic reconnection.

\section{INTRODUCTION}

Microquasars are X-ray binaries with emission dominated by accretion/ejection processes, observed at $\mathrm{X}$-ray and radio wavelengths (Mirabel \& Rodríguez 1999). In microquasars, variability on minute-hour time-scales is investigated since the first observation of short-term radio variability in the black hole system V404 Cyg by Han \& Hjellming (1992). In the black hole candidate GX 339-4, Corbel et al. (2000) reported short-term radio oscillations of $\sim 130 \mathrm{~min}$ at two independent radio frequencies with the higher radio frequency oscillations leading the lower frequency oscillations by few minutes. This radio emission was preceded by X-ray emission by $\sim 167$ min. Tetarenko et al. (2019) showed time lags in the X-ray and radio emission of Cyg X-1 on the order of tens of minutes. In the well-studied source GRS 1915+105, Fender \& Pooley (1998) reported oscillations of period of $26 \mathrm{~min}$ for simultaneous radio and infrared observations with infrared emission leading the radio emission. Mirabel \& Rodríguez (1999) discussed the oscillations at different wavelengths as expanding magnetized

^ E-mail: rsharma@ mpifr-bonn.mpg.de clouds of relativistic particles. At high resolution, Fender et al. (1999) mapped the spatial morphology of the ejection of plasmoids in GRS 1915+105. Between two of the mapped ejections, single dish monitoring showed quasi-periodic oscillations (QPOs) of 20 $40 \mathrm{~min}$. Plasmoids or blobs can originate in events of magnetic reconnection occurring in the jet itself (e.g. Petropoulou et al. 2016; Sironi et al. 2016) or occurring in the accretion disc (Yuan et al. 2009). In one large reconnection region, because of instabilities such as tearing instabilities, multiple reconnection events may occur generating a sequence of flares or QPOs (Massi \& Poletto 2011; Petropoulou et al. 2016). To understand if this phenomenon is observed in the accretion disc or the jet, simultaneous X-ray and radio observations become a diagonostic tool.

LS I $+61^{\circ} 303$ is a radio-emitting high-mass X-ray binary with the $\mathrm{X}$-ray and radio properties typical of accreting black holes with radio jet, i.e. microquasars: the distinctive relationship between the X-ray luminosity and X-ray photon index (Massi et al. 2017), and the characteristic flat radio spectrum (Zimmermann et al. 2015). In LS I $+61^{\circ} 303$, Taylor et al. (1992) observed a step-like pattern in the total radio flux density with time-scale of $1000 \mathrm{~s}$. Peracaula et al. (1997) did the first timing analysis and found microflares as- 

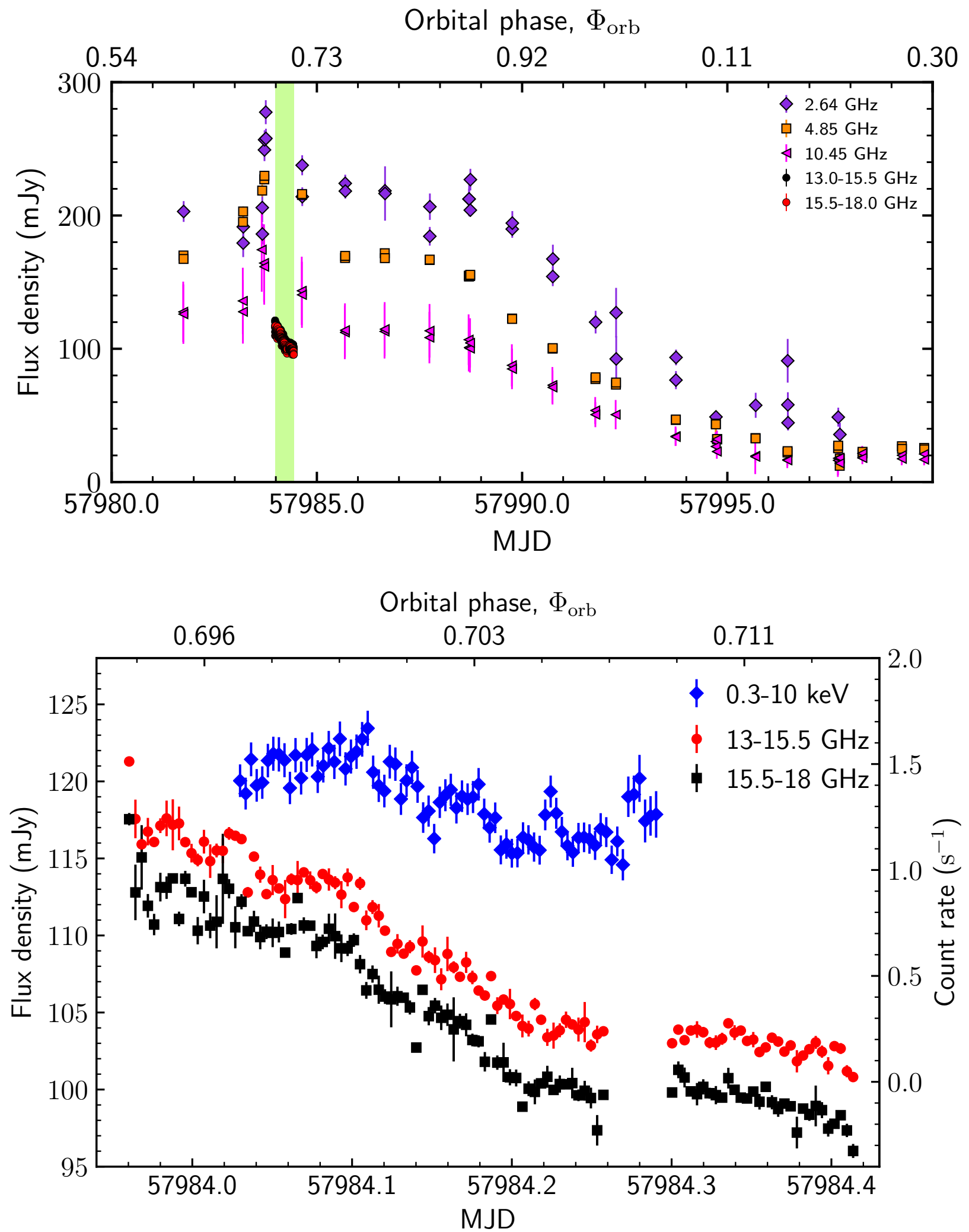

Figure 1. Top: Radio light curves of LS I $+61^{\circ} 303$ as observed with the Effelsberg telescope at 2.64, 4.85, and $10.45 \mathrm{GHz}$ (see Massi et al. 2020). The green-shaded region represents the time interval of observation of LS I $+61^{\circ} 303$ by AMI-LA telescope at $13-15.5$ and $15.5-18$ GHz band, which is clearly during the beginning of the decay of the radio outburst. Bottom: Radio light curve of LS I $+61^{\circ} 303$ as observed with the AMI-LA telescope at frequency band of 13-15.5 and 15.5-18 GHz with units listed on the left vertical axis. Simultaneous X-ray light curve is also shown as observed by the XMM-Newton telescope in the energy range $0.3-10 \mathrm{keV}$ (see right vertical axes). Each data point corresponds to an integration time of $5 \mathrm{~min}$. 
sociated with the early decay of the radio outburst with a period of $1.4 \mathrm{~h}$. Longer time-scale radio QPOs of $15 \mathrm{~h}$ have been detected by Jaron et al. (2017) at three different radio frequencies. In the $\mathrm{X}$-ray regime, Harrison et al. (2000) observed variability on timescales similar to those seen in the radio by Peracaula et al. (1997). Chernyakova et al. (2017) reported variability time-scales of $1000 \mathrm{~s}$ in X-ray emission, which are similar to those observed in radio by Taylor et al. (1992). More recently, Nösel et al. (2018) detected QPOs for both radio $(P \sim 55 \mathrm{~min})$ and X-ray $(P \sim 2.4 \mathrm{~h})$ wavelengths, albeit at different epochs. But the relationship between simultaneous radio and $\mathrm{X}$-ray emission remains unexplored.

To probe this relationship between $\mathrm{X}$-ray and radio emission in the system LS I $+61^{\circ} 303$, the source was observed in $2017 \mathrm{Au}-$ gust at several wavelengths for a month with a sampling rate of hours (Massi et al. 2020), and for a time interval of few hours, it was observed with a high sampling rate of few minutes. In this paper we report on the radio and X-ray results of this high sampling rate monitoring, aimed to investigate the relationship and variability in the radio and X-ray emission of LS I $+61^{\circ} 303$. In Section 2, we describe the new radio and X-ray observations of LS I $+61^{\circ} 303$. In Section 3, we describe the analysis methods employed on the data and in Section 4 we present our results. We conclude and discuss our results in Section 5.

\section{OBSERVATIONS}

LS I $+61^{\circ} 303$ was observed extensively with the Effelsberg 100 $\mathrm{m}$ telescope about every $12 \mathrm{~h}$ from 2017 August 16 until 2017 September 13 (MJD 57981.7436 until MJD 58009.3814) at three frequencies, 2.64, 4.85, and $10.45 \mathrm{GHz}$. The light curves obtained are shown in the top panel of Fig. 1. The bottom axis represents time and the upper axis represents the orbital phase, $\phi_{\text {orb }}=$ $\frac{t-t_{0}}{P_{\text {orb }}}-\operatorname{int}\left(\frac{t-t_{0}}{P_{\text {orb }}}\right)$, where $t_{0}=$ MJD 43366.275 and orbital period $P_{\text {orb }}=26.4960 \pm 0.0028 \mathrm{~d}$ (Gregory 2002). The eccentricity of the source has not been firmly established (see Kravtsov et al. 2020). Extensive study on this data is presented in Massi et al. (2020). Below is the description of the radio and X-ray observations performed at a higher sampling rate.

\subsection{AMI-LA}

Radio observations of the source were performed with the AMILA (Zwart et al. 2008) using the upgraded correlator as described in Hickish et al. (2018). The observations were performed continuously for almost $11 \mathrm{~h}$ from 2017 August 18-19 (MJD 57983.9823 until MJD 57984.4362). The observations were performed about every $1.9 \mathrm{~min}$. The bright point-like source J0228+6721 was observed in an interleaved fashion every $700 \mathrm{~s}$ throughout the observation. The observation was carried out at two frequency bands: 13-15.5 and $15.5-18 \mathrm{GHz}$.

Data reduction was performed using CASA ${ }^{1}$ (McMullin et al. 2007). The standard calibration source $3 \mathrm{C} 286$ was used to set the flux density scale, using the Perley \& Butler (2013a) flux scale together with a correction for the $I+Q$ polarization measured by AMI-LA derived from the polarization measurements from Perley \& Butler (2013b). The interleaved calibration source J0228+6721

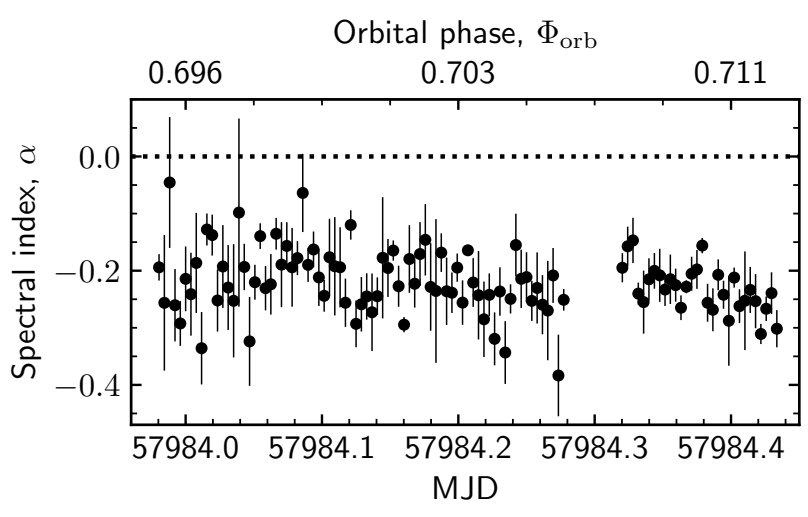

Figure 2. Spectral index variations with time for radio data of $\operatorname{LS~I}+61^{\circ} 303$ obtained with the AMI-LA telescope and integrated every 5 min.

was used for phase calibration, and LS I $+61^{\circ} 303$ was also selfcalibrated in phase assuming a point source model, adequate for the $\approx 30$ arcsec resolution AMI-LA data. An amplitude correction for atmospheric emission and absorption calculated using the 'rain gauge' noise injection system was also applied. After these calibrations, a small spectral index deviation correlating with a change in atmospheric properties was observed on both the calibrator and the source, and was therefore attributed to a change in atmospheric/instrumental properties and this interval between MJD 57984.2772 and MJD 57984.3200 was removed. For comparing with the $X M M-$ Newton observations, as described in the next section, the $5 \mathrm{~min}$ integrated light curves are shown in the bottom panel of Fig. 1 (the red circles and the black squares). The errors reflect the standard deviation. Correlations and timing analysis described in Sections 3 and 4 were performed either on the two independent radio bands or on the flux obtained by fitting over the entire band in order to improve the signal-to-noise ratio.

\section{$2.2 \quad X M M-N e w t o n$}

In this work, we use XMM-Newton observation (Obs. Id. 0795711501) of almost $6 \mathrm{~h}$ taken on 2017 August 19. The X-ray data are simultaneous with the radio data for $\sim 5 \mathrm{~h}$. To analyse it, we used the XMM-Newton Science Analysis software ${ }^{2}$ v.18.0.0. Known hot pixels and electronic noise were removed, and data were filtered to exclude episodes of soft proton flares. The total clean exposure is $\sim 23 \mathrm{ks}$. The light curve was extracted for EPIC-pn camera from a 40 arcsec radius circle centred at the position of LS I $+61^{\circ} 303$ and the background was extracted from a nearby source-free region of 80 arcsec radius. The obtained light curve in the $0.3-10 \mathrm{keV}$ energy range with $5 \mathrm{~min}$ time binning is shown in the bottom panel of Fig. 1 (the blue diamonds).

\section{DATA ANALYSIS}

To study the relationship between the light curves, we analysed them using the discrete correlation function (DCF; Edelson \& Krolik 1988; Hufnagel \& Bregman 1992). This method is used to find correlations and possible time lags in unevenly sampled light 

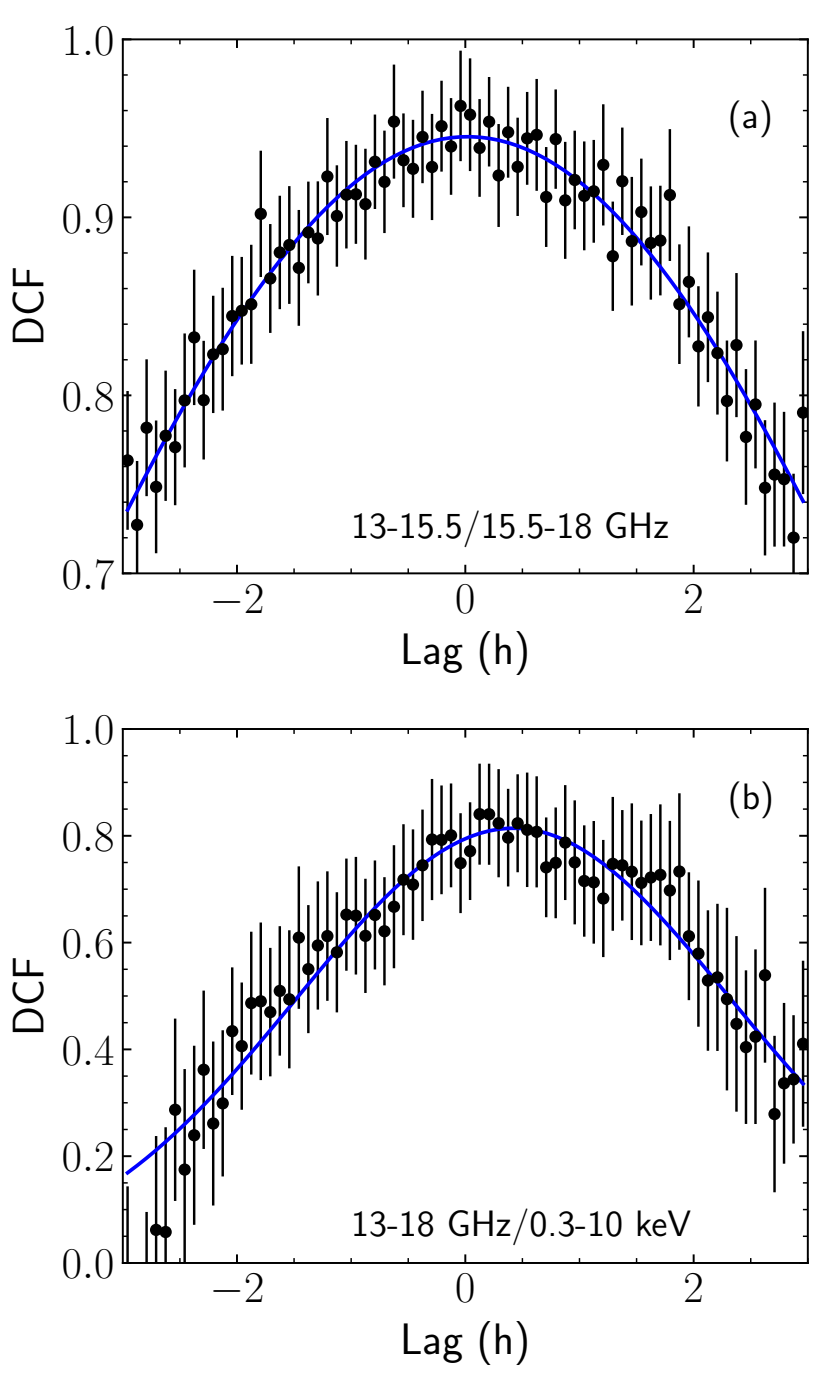

Figure 3. Discrete cross-correlation function of the total flux of (a) 15.5-18 $\mathrm{GHz}$ and $13-15.5 \mathrm{GHz}$ and (b) $13-18 \mathrm{GHz} / 0.3-10 \mathrm{keV}$. The blue curves represent the Gaussian fit to the data with fit parameters given in Table 1. Positive time lag hints that the radio emission precedes the $\mathrm{X}$-ray emission but the lag is consistent with zero within Gaussian dispersion.

curves that is usually the case for astronomical observations and it provides meaningful uncertainty to the correlation coefficients (e.g. Fender et al. 2002; Chidiac et al. 2016; Patiño-Álvarez et al. 2018). Given a time series, $a$ and $b$ with $a(i)$ and $b(j)$ being the individual data points of the time-series, initially the unbinned discrete correlation function (UDCF) is calculated for all possible time lags, $\Delta t_{i j}=t_{j}-t_{i}$ as

$\mathrm{UDCF}_{i j}=\frac{(a(i)-\bar{a})(b(j)-\bar{b})}{\sqrt{\sigma_{a}^{2} \sigma_{b}^{2}}}$,

where $\bar{a}$ and $\bar{b}$ are the respective mean of the time series and $\sigma_{a}^{2}$ and $\sigma_{b}^{2}$ are their variance. The UDCF is then binned in time taking $\tau$ as the centre of the bin and $M$ as the number of pairs in each bin,

$\operatorname{DCF}(\tau)=\frac{1}{M} \sum_{\tau-\Delta \tau / 2}^{\tau+\Delta \tau / 2} \operatorname{UDCF}_{i j}(\tau)$
Table 1. Best-fitting parameters of the Gaussian function fitted to the DCF in Fig. 3 according to Eq. 4. $A$ is the amplitude of the Gaussian, $T$ is the time-lag, and $w$ represents the width. The error in brackets of time-lag $T$ represent the fit errors. Although using a conservative approach, the error on time-lag is given by the Gaussian width, $w$.

\begin{tabular}{lcccc}
\hline Frequency & $A$ & $T(\min )$ & $w(\mathrm{~h})$ & $\chi_{\text {red }}^{2}$ \\
\hline $13-15.5 / 15.5-18 \mathrm{GHz}$ & $0.94 \pm 0.01$ & $1.2[ \pm 1.2]$ & $4.0 \pm 0.1$ & 0.2 \\
$13-18 \mathrm{GHz} / 0.3-10 \mathrm{keV}$ & $0.81 \pm 0.01$ & $25.2[ \pm 2.4]$ & $1.9 \pm 0.1$ & 0.3 \\
\hline
\end{tabular}

The error for each bin is given by

$\sigma_{D C F}(\tau)=\frac{1}{M-1} \sqrt{\sum_{\tau-\Delta \tau / 2}^{\tau+\Delta \tau / 2}\left[\mathrm{UDCF}_{i j}-\operatorname{DCF}(\tau)\right]^{2}}$.

The normalisation (mean and standard deviation of respective light curves) assumes that the light curves are statistically stationary, which is not true for variable data. To make the light curve stationary, the mean and standard deviation are calculated using only the points that overlap in a given time-lag bin, thus making the DCF bound between $[-1,+1]$ interval (White \& Peterson 1994). Also as mentioned in Edelson \& Krolik (1988), to keep the normalisation correct, we need to omit the zero lag pairs (i.e. pairs where $i=j$ ). For a more quantitative study, we performed least-squares fit of a Gaussian to the DCF profiles. Thus, to estimate the time-lag between emission at two frequencies, a Gaussian function of the following form is fit to the DCF:

$\operatorname{DCF}(t)=A \times \exp \left[\frac{-(t-T)^{2}}{2 w^{2}}\right]$,

where $A$ is the peak DCF value, $T$ is the time-lag at peak of DCF, and $w$ is the width of the Gaussian function. As a conservative approach we use the width, $w$ as an error on the time-lag (see Alexander 2013; Chidiac et al. 2016).

In Fig. 1 (bottom panel), we note that there are small variability of few $\mathrm{mJy}$ and counts $\mathrm{s}^{-1}$ superimposed over the long-term decaying trend of the radio and X-ray light curves, respectively. The analysis of these small variations require the removal of the long-term trend from the light curves (e.g. see Fender et al. 2002; Jaron et al. 2017). In order to remove the long-term trend from the data, we subtraced a piecewise linear function from the data. For the radio light curves, we fit the linear function first from MJD 57983.9823 to MJD 57984.2772 and then from MJD 57984.3200 to MJD 57984.4362. For the XMM data, the fit was applied to the whole interval.

In order to find the statistical significance of the variability, we calculate the fractional variability (Vaughan et al. 2003) of the detrended data as

$F_{\mathrm{var}}=\sqrt{\frac{S^{2}-\overline{\sigma_{\mathrm{err}}^{2}}}{\bar{x}^{2}}}$,

where $S^{2}$ is the variance of the light curve, $\overline{\sigma_{\text {err }}^{2}}$ is the mean square error, and $\bar{x}^{2}$ is the mean flux of the light curve. The error in the fractional variability is estimated as

$\operatorname{err}\left(F_{\mathrm{var}}\right)=\sqrt{\frac{1}{2 N}\left(\frac{\overline{\sigma_{\mathrm{err}}^{2}}}{F_{\mathrm{var}} \times \bar{x}^{2}}\right)^{2}+\frac{\overline{\sigma_{\mathrm{err}}^{2}}}{N \times \bar{x}^{2}}}$.

The detrended data were then analysed using the Lomb-Scargle periodogram, which is a powerful tool to find and test the significance of weak periodic signals in unevenly sampled data (Lomb 

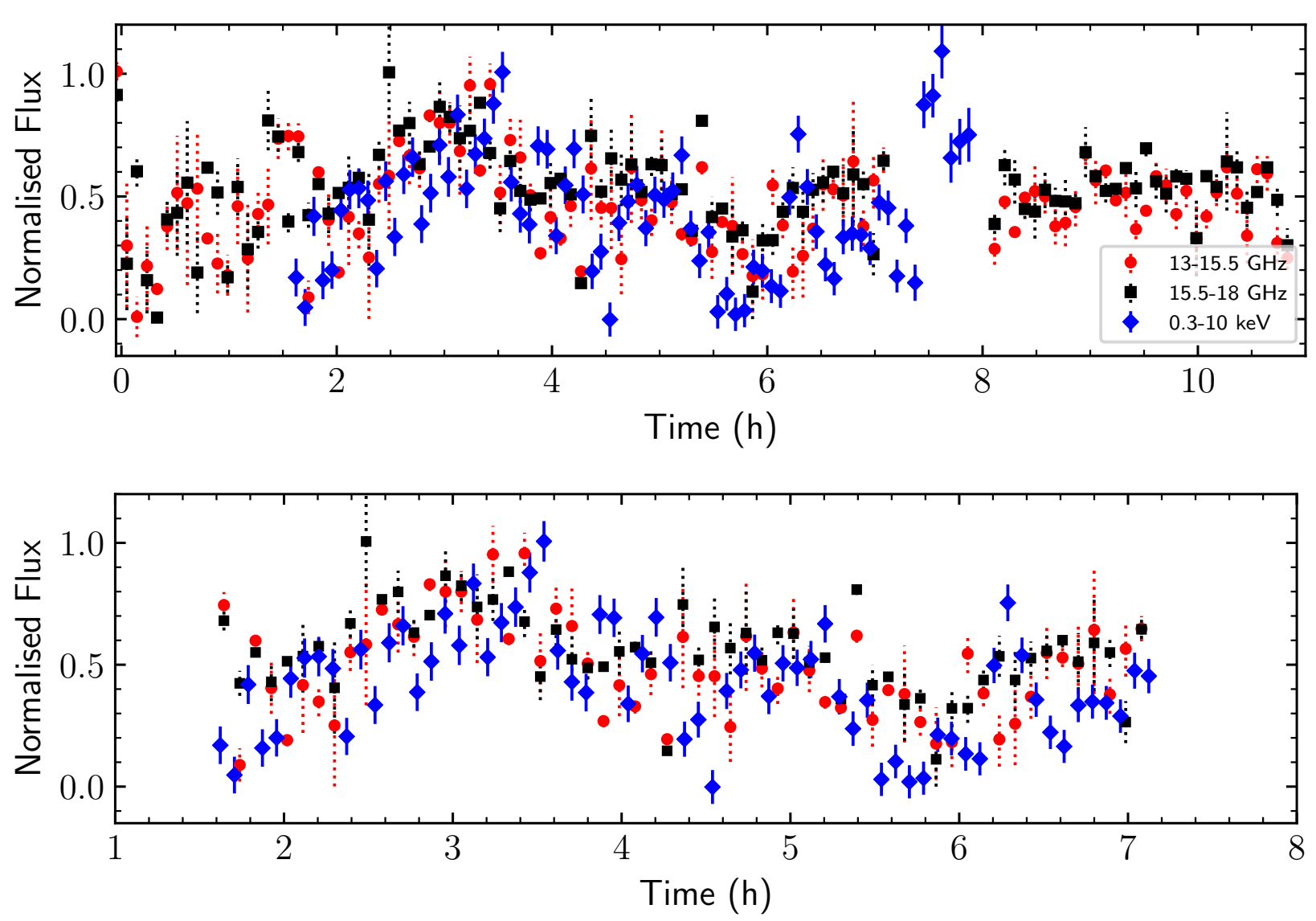

Figure 4. Detrended and normalized light curves for comparison. Top: For the entire duration of the observations of about $11 \mathrm{~h}$ with $13-15.5 \mathrm{GHz}$, 15.5-18 $\mathrm{GHz}$, and $0.3-10 \mathrm{keV}$ represented by the red circles, the black squares, and the blue diamonds, respectively. Bottom: Zoom-in of the light curves only for the simultaneous radio and $\mathrm{X}$-ray observations of $5 \mathrm{~h}$.

1976; Scargle 1982). Fischer-randomization test is used to test the significance of the found periodic signal, where the flux is permuted thousand times and thousand new randomized time series are created and their periodograms calculated (Linnell Nemec \& Nemec 1985). The proportion of permuted time series that contain a higher peak in the periodogram than the original periodogram at any frequency then gives the false alarm probability, $p$ of the peak. If $p<0.01$, the period is significant and if $0.01<p<0.1$ the period is marginally significant. In order to check if the found period is a true period and it is not generated due to sampling, the sampling function is analysed. The flux density are assigned a constant value equal to one. This function is further analysed using the Fourier transform.

\section{RESULTS}

The radio and X-ray light curves are shown in the bottom panel of Fig. 1. We see that the radio emission displays a decaying trend with the flux density decreasing from around $120 \mathrm{mJy}$ to $95 \mathrm{mJy}$ in $\sim 11 \mathrm{~h}$ observation. In fact, as shown in the top panel of Fig. 1, the observation with the AMI-LA telescope took place during the beginning of the decay of the large radio outburst of LS I $+61^{\circ} 303$. The X-ray light curve also shows a general decaying trend in its counts $\mathrm{s}^{-1}$. Figure 2 shows the radio spectral index, $\alpha=\log \left(S_{1} / S_{2}\right) / \log \left(v_{1} / v_{2}\right)$, which is negative throughout the $11 \mathrm{~h}$ observation. In this section, we first discuss the results of the correlation analysis of the simultaneous radio and X-ray data and then of the variability.

To find the strength of association between the two time series, we calculated the Spearman correlation $\left(r_{s}\right)$. For 13-15.5/15.5$18 \mathrm{GHz}, r_{s}=0.94$, for $13-15.5 \mathrm{GHz} / 0.3-10 \mathrm{keV}, r_{s}=0.84$ and for $15.5-18 \mathrm{GHz} / 0.3-10 \mathrm{keV}, r_{s}=0.83$. All correlations have a $\mathrm{p}-$ value $<0.001$ which means that it is very unlikely to get this result by chance. Squaring the coefficients for the radio/X-ray data yields the strength of correlation $r_{\mathrm{s}}^{2} \sim 0.7$, i.e. 70 per cent of the variability in one frequency is due to the variability in the other frequency.

To further quantify this, we also compute the discrete cross-correlation functions (DCF). Figure 3(a) shows the crosscorrelation between the two radio bands, i.e. 13-15.5 and 15.5-18 GHz. The blue curve represents the Gaussian fit to the data with fit parameters given in Table 1 . We see that the two frequency bands are strongly correlated up to 94 per cent with zero time-lag. Crosscorrelations between simultaneous radio and X-ray data are shown in Fig. 3(b). We find that the radio band is strongly correlated with the X-ray. The DCF peaks at 81 per cent with a time-lag of $\sim 25 \mathrm{~min}$ and a conservative error estimate of $\sim 2 \mathrm{~h}$.

To further analyse the data, we work with the detrended light curves. The fractional variability amplitude of the radio frequencies is $\sim 64.0 \pm 0.2$ per cent and that of the $\mathrm{X}$-ray is $\sim 15.0 \pm 5.0$ per cent. The normalized detrended flux for both the radio and X-ray emis- 

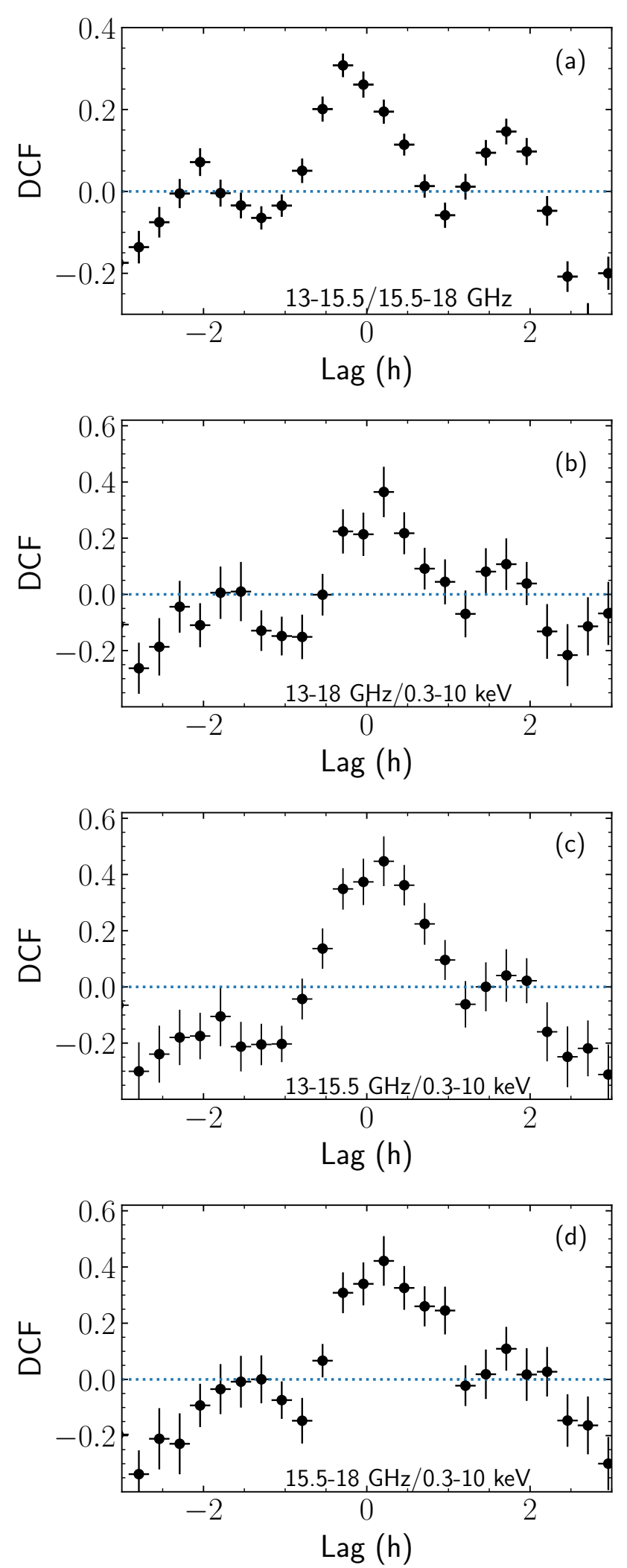

Figure 5. Discrete cross-correlation of detrended light curves of (a) 15.5-18 and $13-15.5 \mathrm{GHz}$ with secondary peaks representing possible periodicity, (b) $13-18 \mathrm{GHz} / 0.3-10 \mathrm{keV}$, (c) $13-15.5 \mathrm{GHz} / 0.3-10 \mathrm{keV}$, and (d) $15.5-$ $18 \mathrm{GHz} / 0.3-10 \mathrm{keV}$. All correlations peak around zero time-lag. The bluedotted line represents correlation coefficient of zero.
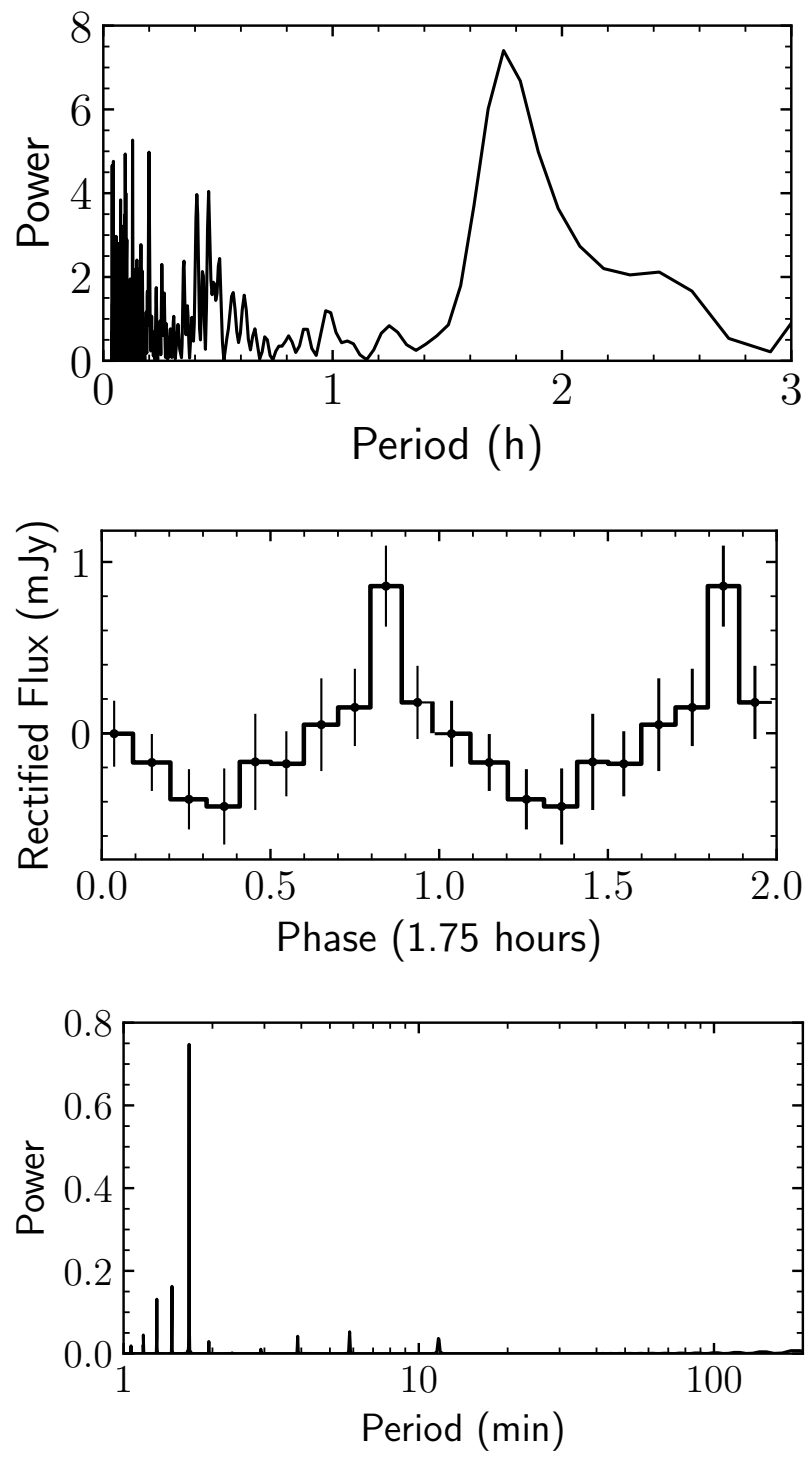

Figure 6. Top: Lomb-Scargle periodogram of $13-18 \mathrm{GHz}$ detrended radio data showing a significant peak at $1.75 \pm 0.10 \mathrm{~h}$. Middle: Phase-averaged data folded with the significant period of $1.75 \mathrm{~h}$ in 10 bins. For clarity, the data are repeated in the second cycle. Bottom: Fourier transform of the sampling function of radio data showing periodic feature with maximum power at a period of $1.7 \mathrm{~min}$ and no feature at $105 \mathrm{~min}(1.75 \mathrm{~h})$. The $\mathrm{x}$-axis is in $\log$ scale.

sion (normalized with respect to the maximum flux density in their respective light curves) are shown in the top panel of Fig. 4 and a zoom-in of the same for the $5 \mathrm{~h}$ simultaneous observations is shown in the bottom panel of Fig. 4. There is variability with varying amplitude. Qualitatively, the variability at both radio frequencies seems to coincide with the variability in the X-rays (see Fig. 4). We perform the cross-correlation analysis using the simultaneous $\mathrm{X}$ ray and radio data of the detrended light curves. Figure 5(a) shows the cross-correlation between the two radio bands. The DCF peaks at around zero time lag with a coefficient of $0.30 \pm 0.04$. There is also a correlation at negative time lag of $2.0 \pm 0.1 \mathrm{~h}$ and positive time lag of $1.7 \pm 0.1 \mathrm{~h}$ with DCF coefficients of $0.10 \pm 0.04$ and 
$0.13 \pm 0.04$, respectively. These secondary peaks hint at a possible periodicity in the radio data. The cross-correlation between the detrended radio and X-ray data is shown in Fig. 5(b). The DCF of radio band with $\mathrm{X}$-ray peaks around zero lag with a coefficient of $0.36 \pm 0.10$. The same DCF pattern is also evident when we correlate the X-ray data with the two independent radio bands (Figs $5 \mathrm{c}$ and d). The DCF of both radio bands with X-ray peaks around zero lag with a coefficient of $0.45 \pm 0.10$ and $0.42 \pm 0.10$ for $13-15.5$ $\mathrm{GHz} / 0.3-10 \mathrm{keV}$ and $15.5-18 \mathrm{GHz} / 0.3-10 \mathrm{keV}$, respectively.

As mentioned earlier, the radio and $\mathrm{X}$-ray light curves show variability. The $5 \mathrm{~h}$ simultaneous observations of radio and $\mathrm{X}$ ray data do not reveal significant features in the Lomb-Scargle periodogram. Therefore, we further use the full $11 \mathrm{~h}$ radio data. Top panel of Fig. 6 shows the Lomb-Scargle periodogram for 13$18 \mathrm{GHz}$ radio band, with a dominant and significant peak at 1.75 $\pm 0.10 \mathrm{~h}$, corresponding to the secondary peaks in the DCFs. The phase-averaged data folded with a period of $1.75 \mathrm{~h}$ are shown in the middle panel of Fig. 6. As shown in the bottom panel of Fig. 6, we see a dominant feature in the sampling function at about $1.7 \mathrm{~min}$, i.e. the sampling rate. No feature is present at the found period of $105 \mathrm{~min}$ (i.e. $1.75 \mathrm{~h}$ ) due to which we know that the found period is not an effect of the sampling. This confirms that the radio frequency has a QPO of $1.75 \pm 0.10 \mathrm{~h}$.

\section{CONCLUSIONS AND DISCUSSION}

We observed LS I $+61^{\circ} 303$ with the AMI-LA telescope for around $11 \mathrm{~h}$ at two radio frequency bands, $13-15.5$ and $15.5-18 \mathrm{GHz}$. We also observed it with the XMM-Newton telescope for about $6 \mathrm{~h}$ at energy range of $0.3-10 \mathrm{keV}, 5 \mathrm{~h}$ of which was simultaneous with the radio observations. Both radio and X-ray show variability superimposed over the long-term trend.

We find that the radio and X-ray emission are correlated up to 81 per cent. In the detrended light curves, i.e. after the removal of the long-term trend, the variability in X-ray and radio emission are correlated up to 40 per cent. The radio variability overlaps with the X-ray variability. The DCF analysis hints that the radio emission leads the X-ray emission by $\sim 25 \mathrm{~min}$, but within Gaussian dispersion they have zero time-lag. The better signal-to-noise ratio and longer observation time interval of radio data allow us to establish by Lomb-Scargle periodogram QPOs with a period of $1.75 \pm 0.10 \mathrm{~h}$ in $\mathrm{LS} \mathrm{I}+61^{\circ} 303$.

The significant radio/X-ray correlation implies that both the radio and X-ray emission are due to the same electron population. But are these emissions due to the same or different physical processes? Our simultaneous radio/X-ray observations were performed during the beginning of the decay of a major radio outburst interlapsed by optically thin flares (also see Massi et al. 2020). Optically thin outbursts in microquasars are explained due to shocks and/or magnetic reconnection events (Romero et al. 2017 and references therein).

Multiple reconnection events lead to multiple ejection of plasmoids of different size, whose period can vary from minutes, hours to days (Petropoulou et al. 2016; Sironi et al. 2016). On the basis of these models, the magnetic reconnection and consequent formation of plasmoids can occur at two locations in X-ray binaries. The first location is magnetic reconnection in the accretion disc (Yuan et al. 2009) and the consequent travelling of plasmoids into the jet. Mirabel et al. (1998) showed that in GRS 1915+105 the ejection of electrons from the accretion disc were first observed in the X-rays, then in the infrared and finally in the radio band.
These kind of observations, interpreted as accretion disc ejections, are expected during the onset of an outburst and not during its decay. Infact, in Nösel et al. (2018), we studied the location of QPOs along the orbit of LS I $+61^{\circ} 303$. Statistically, it was found that the $\mathrm{X}$-ray QPOs are present during the onset of the steady radio jet. Possibly those X-ray variability were associated with ejection of plasmoids from the accretion disc. Other than the accretion disc, the magnetic reconnection events can also occur in the jet itself (Petropoulou et al. 2016; Sironi et al. 2016). In the jets, radio emission is due to the synchrotron process, with relativistic plasma injected in quasi-periodic fashion. However, X-ray emission can be either due to synchrotron emission (Markoff et al. 2005) or due to up-scattering of the low-energy radio photons via inverse Compton scattering, i.e. synchrotron self-Compton (SSC) (e.g. fig. 3 in Romero et al. 2017). If the X-ray emission leads the radio emission, it would imply a synchrotron origin and if the radio emission leads the X-ray emission, it would be due to SSC scattering. A similar phenomenon, i.e. the radio emission leading the $\mathrm{X}$-ray emission by about 2 weeks, was observed in the blazar PKS 1510-089 by Marscher et al. (2003) and explained as SSC emission (Marscher et al. 2010 and references therein). In our observations, the found time-lag between the radio and X-ray emission hints that the radio emission leads the X-ray emission but within Gaussian dispersion it could have zero time-lag. Future sensitive observations covering longer time intervals are clearly mandatory to confirm which physical process dominates at these frequencies.

\section{ACKNOWLEDGEMENTS}

The authors would like to thank the referee for their valuable comments and Gisela Ortiz-Leon for carefully reading the manuscript. RS thanks V. M. Patiño-Álvarez and Céline Chidiac for discussion on DCF. We thank the staff of the Mullard Radio Astronomy Observatory, University of Cambridge, for their support in the maintenance and operation of AMI. We acknowledge support from the European Research Council under grant ERC-2012StG-307215 LODESTONE. Based on observations obtained with $X M M-N e w t o n$, an ESA science mission with instruments and contributions directly funded by ESA Member States and NASA. The authors acknowledge support by the state of Baden-Württemberg through bwHPC. This work was supported by DFG through the grant MA 7807/2-1.

\section{DATA AVAILABILITY}

The data underlying this article will be shared on reasonable request to the corresponding author.

\section{REFERENCES}

Alexander T., 2013, arXiv e-prints, p. arXiv:1302.1508

Chernyakova M., Babyk I., Malyshev D., Vovk I., Tsygankov S., Takahashi H., Fukazawa Y., 2017, MNRAS, 470, 1718

Chidiac C., et al., 2016, A\&A, 590, A61

Corbel S., Fender R. P., Tzioumis A. K., Nowak M., McIntyre V., Durouchoux P., Sood R., 2000, A\&A, 359, 251

Edelson R. A., Krolik J. H., 1988, ApJ, 333, 646

Fender R. P., Pooley G. G., 1998, MNRAS, 300, 573

Fender R. P., Garrington S. T., McKay D. J., Muxlow T. W. B., Pooley G. G., Spencer R. E., Stirling A. M., Waltman E. B., 1999, MNRAS, 304,865 


\section{$8 \quad$ R. Sharma et al.}

Fender R. P., Rayner D., Trushkin S. A., O'Brien K., Sault R. J., Pooley G. G., Norris R. P., 2002, MNRAS, 330, 212

Gregory P. C., 2002, ApJ, 575, 427

Han X., Hjellming R. M., 1992, ApJ, 400, 304

Harrison F. A., Ray P. S., Leahy D. A., Waltman E. B., Pooley G. G., 2000, ApJ, 528, 454

Hickish J., et al., 2018, MNRAS, 475, 5677

Hufnagel B. R., Bregman J. N., 1992, ApJ, 386, 473

Jaron F., Sharma R., Massi M., Fuhrmann L., Angelakis E., Myserlis I., Li G.-X., Shi X., 2017, MNRAS, 471, L110

Kravtsov V., et al., 2020, arXiv e-prints, p. arXiv:2010.00999

Linnell Nemec A. F., Nemec J. M., 1985, AJ, 90, 2317

Lomb N. R., 1976, Ap\&SS, 39, 447

Markoff S., Nowak M. A., Wilms J., 2005, ApJ, 635, 1203

Marscher A. P., et al., 2003, in High Energy Blazar Astronomy. p. 173

Marscher A. P., et al., 2010, ApJ, 710, L126

Massi M., Poletto G., 2011, Mem. Soc. Astron. Italiana, 82, 145

Massi M., Migliari S., Chernyakova M., 2017, MNRAS, 468, 3689

Massi M., et al., 2020, MNRAS, 498, 3592

McMullin J. P., Waters B., Schiebel D., Young W., Golap K., 2007, in Shaw R. A., Hill F., Bell D. J., eds, Astronomical Society of the Pacific Conference Series Vol. 376, Astronomical Data Analysis Software and Systems XVI. p. 127

Mirabel I. F., Rodríguez L. F., 1999, ARA\&A, 37, 409

Mirabel I. F., Dhawan V., Chaty S., Rodriguez L. F., Marti J., Robinson C. R., Swank J., Geballe T., 1998, A\&A, 330, L9

Nösel S., Sharma R., Massi M., Cimò G., Chernyakova M., 2018, MNRAS, 476, 2516

Patiño-Álvarez V. M., et al., 2018, MNRAS, 479, 2037

Peracaula M., Marti J., Paredes J. M., 1997, A\&A, 328, 283

Perley R. A., Butler B. J., 2013a, ApJS, 204, 19

Perley R. A., Butler B. J., 2013b, ApJS, 206, 16

Petropoulou M., Giannios D., Sironi L., 2016, MNRAS, 462, 3325

Romero G. E., Boettcher M., Markoff S., Tavecchio F., 2017, Space Sci. Rev.,

Scargle J. D., 1982, ApJ, 263, 835

Sironi L., Giannios D., Petropoulou M., 2016, MNRAS, 462, 48

Taylor A. R., Kenny H. T., Spencer R. E., Tzioumis A., 1992, ApJ, 395, 268

Tetarenko A. J., Casella P., Miller-Jones J. C. A., Sivakoff G. R., Tetarenko B. E., Maccarone T. J., Gand hi P., Eikenberry S., 2019, MNRAS, 484, 2987

Vaughan S., Edelson R., Warwick R. S., Uttley P., 2003, MNRAS, 345, 1271

White R. J., Peterson B. M., 1994, PASP, 106, 879

Yuan F., Lin J., Wu K., Ho L. C., 2009, MNRAS, 395, 2183

Zimmermann L., Fuhrmann L., Massi M., 2015, A\&A, 580, L2

Zwart J. T. L., et al., 2008, MNRAS, 391, 1545

This paper has been typeset from a $\mathrm{T}_{\mathrm{E}} \mathrm{X} / \mathrm{L} \mathrm{T}_{\mathrm{E}} \mathrm{X}$ file prepared by the author. 